Document downloaded from:

http://hdl.handle.net/10251/36787

This paper must be cited as:

Micó Ruiz, JC.; Soler Fernández, D. (2011). The capacitated general windy routing problem with turn penalties. Operations Research Letters. 39(4):265-271. doi:10.1016/j.orl.2011.04.007.

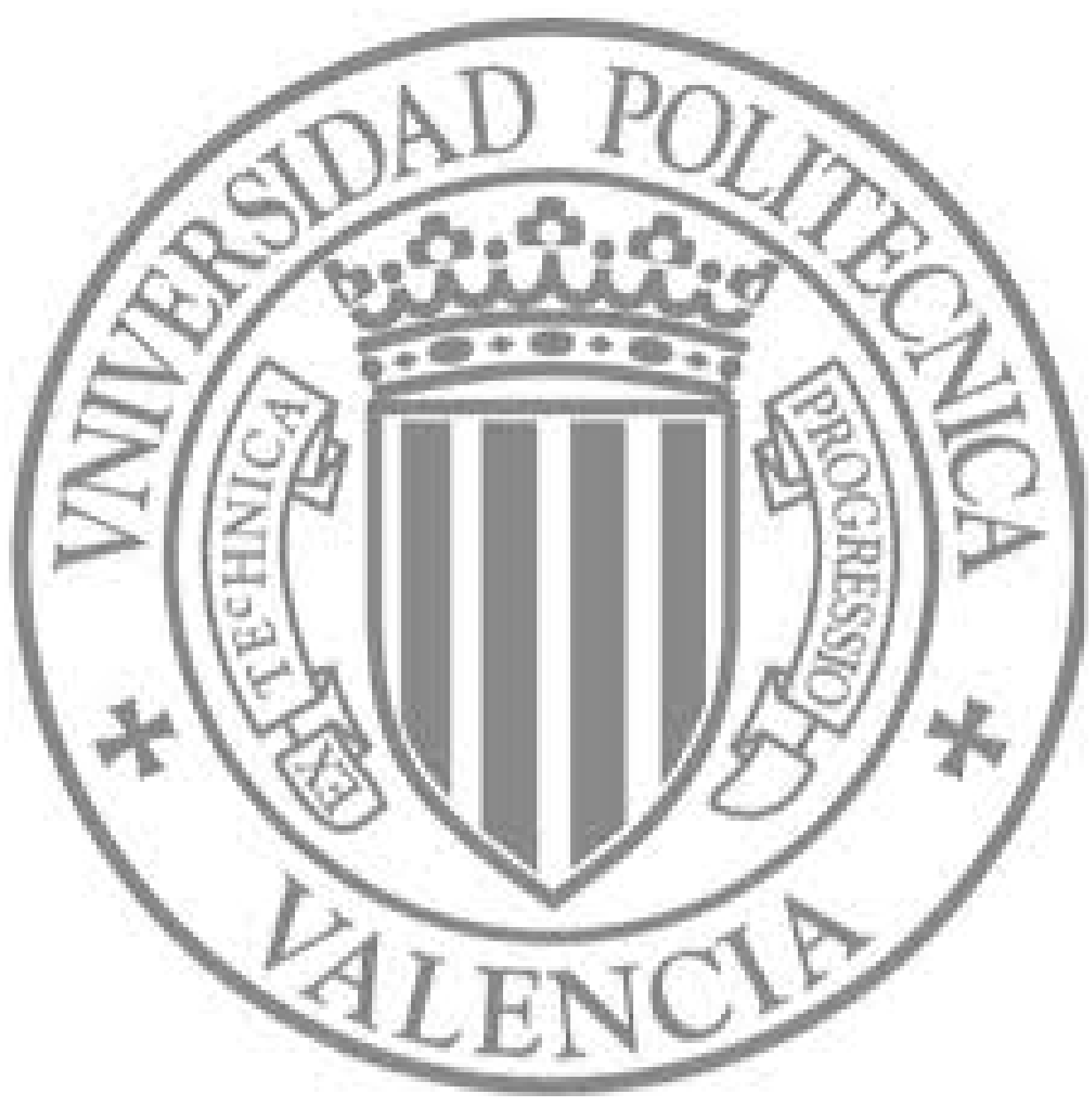

The final publication is available at

http://dx.doi.org/10.1016/j.orl.2011.04.007

Copyright Elsevier 


\title{
The capacitated general windy routing problem with turn penalties
}

\author{
Joan C. Micó, David Soler* \\ Institut Universitari de Matemàtica Pura i Aplicada. \\ Universitat Politècnica de València. València, Spain
}

\begin{abstract}
In this paper we present the Capacitated General Windy Routing Problem with Turn Penalties. This new problem subsumes many important and wellknown arc and node routing problems, and it takes into account turn penalties and forbidden turns, which are crucial in many real-life applications, particularly in downtown areas and for large-size vehicles. We provide a way to solve this problem both optimally and heuristically by transforming it into a Generalized Vehicle Routing Problem.
\end{abstract}

Keywords: Vehicle routing, turn penalties, transformation.

\section{Introduction}

Arc and node routing problems consist basically of finding a set of routes covering certain arcs, edges and/or vertices of a graph, which meet certain conditions. These problems allow us to model and to solve many real-life problems, especially in the context of transportation, distribution management and scheduling, like waste collection, mail delivery, snow removal, pick-up or delivery of any kind of goods, network

* Corresponding author: David Soler, Institut Universitari de Matemàtica Pura i Aplicada. Universitat Politècnica de València. Camí de Vera s/n, 46022 València, Spain. e-mail: dsoler@mat.upv.es. 
maintenance, etc. Hundreds of papers have been written in the last decades about different kinds of arc and/or node routing problems, mainly based on the type of graph (undirected, directed or mixed), on the number of vehicles (a single vehicle or a fleet of vehicles) and on the type of elements to be covered (links, vertices or both).

Very recently, [1] has introduced a general problem that subsumes several wellknown arc and node routing problems. This problem is called the Capacitated General Windy Routing Problem (CGWRP) and can be stated as follows:

Let $G=(V, E)$ be an undirected graph, $V=\{0,1, \ldots, n\}$ being its set of vertices, where 0 is a depot, and $E$ being the edge set, where edge $e \in E$ is often represented by an ordered pair of vertices $(i, j)$ with $i<j$.

$A$ subset $V_{R}$ of required vertices (including 0 ) and a subset $E_{R}$ of required edges are considered. A non-negative demand $q_{i}$ is associated with each required vertex $i$ (except for vertex 0) and two non-negative demands $q_{i j}$ and $q_{j i}$ are associated with each required edge $(i, j)$, one for each direction of traversal. Moreover, two non-negative costs $c_{i j}$ and $c_{j i}$ are associated with each edge, one for each direction of traversal. Finally, a fleet of $k$ vehicles with the same capacity $W$ is available at the depot.

Find a least-cost set of $k$ routes, each starting and ending at the depot, such that all required vertices and edges are visited, each vertex demand is satisfied by only one vehicle, for each required edge one and only one of its demands is satisfied by only one vehicle, and the total demand of each route does not exceed $W$.

The term "general" means that both, edges and vertices, can be required, while the term "windy" refers to the fact that the cost of traversing an edge depends on the direction of traversal, as with or against the wind. Moreover, if one of the two costs of an edge is infinite, the edge can be replaced by an arc, and if both costs are equal, we have an edge as defined in the conventional theory. Therefore, this definition generalizes many undirected, directed and mixed arc and node routing problems.

As examples of well-known particular cases of the CGWRP, we can mention:

- The Traveling Salesman Problem (TSP) in its undirected version. It occurs when $E_{R}=\emptyset, V_{R}=V, k=1$ and the two costs associated with each edge are equal. Note that when $k=1$ it is assumed that the vehicle can satisfy all demand, and therefore, 
it is not necessary to associate demands with required vertices.

- The Chinese Postman Problem (CPP) in its undirected version. It occurs when $E_{R}=E, V_{R}=\emptyset, k=1$, the two costs associated with each edge are equal and the two demands associated with each edge are equal. As in the previous case, $k=1$, therefore, no demand is associated with the required edges.

- The Capacitated Arc Routing Problem (CARP) in its undirected version. It occurs when $V_{R}=\emptyset, k>1$, the two costs associated with each edge are equal and the two demands associated with each edge are equal.

- The Asymmetric Capacitated Vehicle Routing Problem (ACVRP). It occurs when $V_{R}=V, k>1, E_{R}=\emptyset$ and one cost of each edge is infinite. This case will be essential to solve the problem studied in this paper. Figure 1 shows the relationship among most of the routing problems cited in this section.

Paper [1] also presents a transformation of the CGWRP into a Generalized Vehicle Routing Problem (GVRP), a problem introduced in [11] that allows us to model reallife problems in which each customer has several alternative service locations, and only one of them has to be selected for service. As the GVRP is also essential in this paper, we need to formally define it:

Let $G=(V, A)$ be a directed graph where the vertex set $V$ is partitioned into $m+1$ nonempty subsets $S_{0}, S_{1}, \ldots, S_{m}$ such that $S_{0}$ has only a vertex (the depot), $S_{h}$ ( $h=$ $1, \ldots, m)$ has $l_{h}$ vertices, each with its own non-negative demand, and each arc $(i, j) \in A$ has a cost $c_{i j} \geq 0$ associated with it. Moreover, a fleet of $k$ vehicles having the same capacity $W$ is available at the depot. Find a least-cost set of $k$ routes, each starting and ending at the depot, such that each subset $S_{h}(h=1, \ldots, m)$ is visited exactly once and the sum of the demands of every route does not exceed the capacity $W$ of the vehicle.

Note that this definition is taken from [1], but in other papers $([11,16])$ the GVRP definition requires that all vertices corresponding to the same subset $S_{h}$ have the same demand, that is, each $S_{h}$ represents $l_{h}$ possible locations of the same customer.

As far as we know, no specific algorithm has been developed for the GVRP. However, if all vertices corresponding to the same subset $S_{h}$ have the same demand, the GVRP can be solved by transforming it into an ACVRP ([16]). As the literature provides 
both exact and heuristic procedures to solve the ACVRP (see e.g. $[9,10,13,17]$ ), at least from a theoretical point of view, this particular case of the GVRP can be solved.

Note that in [1] the CGWRP is transformed into a GVRP where vertices corresponding to the same subset $S_{h}$ may have different demands. But in the particular case of the CGWRP in which $q_{i j}=q_{j i}$ for each required edge $(i, j)$, the transformation gives place to a GVRP with the same demand for all vertices in the same subset $S_{h}$ and therefore, the result given in [1] is useful to solve real-world problems. It seems that the symmetric case of the edge demands fits many more real capacitated vehicle routing problems than the asymmetric one, at least according to the literature, where we have not been able to find any paper about real-world capacitated vehicle routing problems with direction-dependent demands. In fact, the authors say in [1] that "the idea of introducing direction-dependent demands is new", and in contrast, the literature is full of articles on real-world routing problems where the link demands do not depend on the direction of traversing the link, such as those solving waste collection or mail delivery problems (see e.g. $[3,6,14]$ ), which can be extended to the windy case.

From now on, by CGWRP-ed (equal demand) we will understand the particular case of the CGWRP in which $q_{i j}=q_{j i}$ for each required edge $(i, j)$, we will use the GVRP definition given in $[11,16]$, and therefore, by demand $q_{h}$ associated to $S_{h}$ in a GVRP, we will understand that all vertices in $S_{h}$ have the same demand $q_{h}$.

On the other hand, in many real-life vehicle routing problems, especially those involving downtown areas or large-size vehicles, it is important to consider some kind of penalties associated with the turns. Moreover, some turns, especially U-turns and left turns, can be forbidden. Actually the latter are quite normal in big cities and then, a vehicle route generated through a classical graph model may be illegal and dangerous if it does not respect the traffic signs.

In the last two decades some papers have been written with the aim of extending well-known single vehicle routing problems to consider the existence of turn penalties and forbidden turns (see $[5,7,8,15]$ ).

Early papers considering turn penalties in routing problems with a fleet of vehicles focused on solving real-life applications (see e.g. [6, 14]). Later, turn penalties have been considered in the context of the mixed CARP (MCARP), which is a CARP defined 
on a graph with both arcs and edges, see e.g. [2, 4]. More recently, some authors have taken into account turn penalties or forbidden turns to model real-life routing problems in the context of waste collection ([3]) or snow plowing ([12]).

In this work we deal with an extension of the CGWRP-ed that considers turn penalties and forbidden turns. Following previous papers, we call the new problem the Capacitated General Windy Routing Problem with Turn Penalties (CGWRPTP). In this way, we present a problem that generalizes all the previous works on routing problems with turn penalties, both with a single vehicle and with a fleet of vehicles and many arc and node routing problems studied in the literature. To solve the CGWRPTP we polynomially transform it into a GVRP, which in turn can be transformed, as mentioned above, into an ACVRP (see Figure 1). Note that the CGWRPTP assumes that for each required edge, demands in both directions are equal.

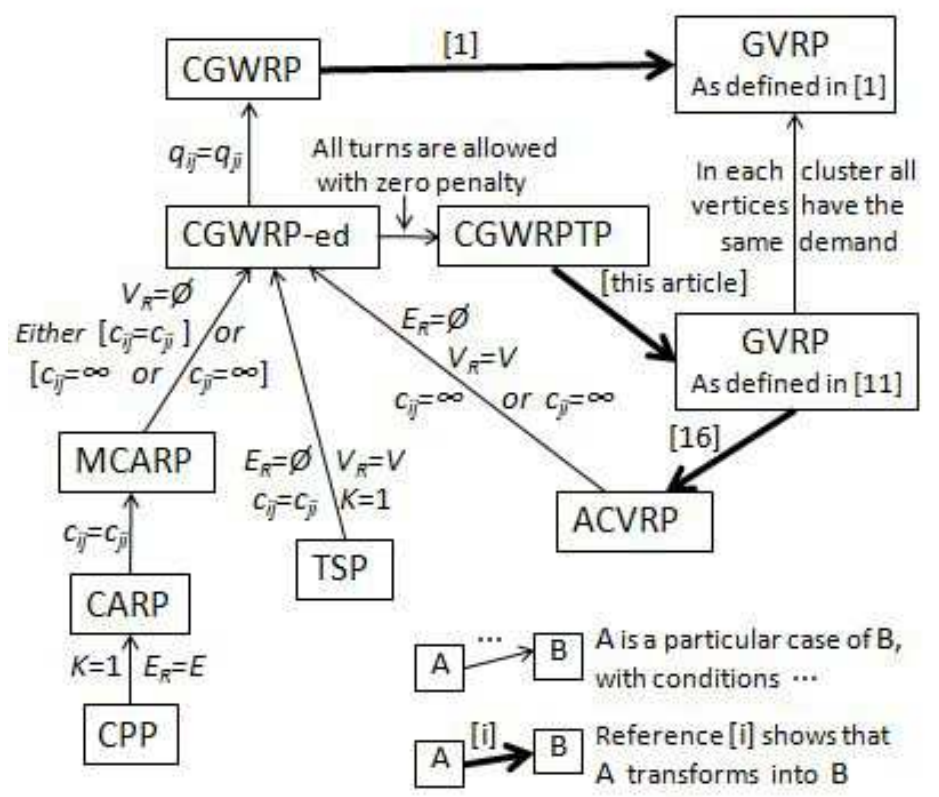

Fig. 1. Relationship among routing problems cited in this section.

The CGWRPTP can approach more closely certain real-world capacitated vehicle routing problems than some of its particular cases already studied. For example, if we think about waste collection in cities located in mountain areas (as is the case in Spain, a mountainous country which has many cities of this kind, even on top of mountains, for the historical sake of better defense and views of the surrounding area), many streets are steep, therefore, it makes sense to consider different costs for both directions of 
traversal. Moreover, in historical city centers mainly, streets are also narrow, making it difficult to maneuver the waste collection trucks at street crossings. All U-turns are likely to be forbidden, and many allowed turns are dangerous or at least difficult. As a result, it makes sense to consider also turn penalties.

Our transformation can be considered an extension to the windy and multivehicle case of the transformation given in [15], and somehow, an extension to turn penalties and forbidden turns of the transformation given in [1]. In fact, the problems studied in these two papers can be solved through our transformation and in both cases, our transformed graph has the same structure than those presented in the two cited papers except for the arc costs. The latter is due to the different ways of treating the required edges and defining paths between arcs.

This paper is organized as follows. Section 2 formally defines the CGWRPTP. Section 3 shows the transformation of the CGWRPTP into a GVRP, which is illustrated with an example, and it explains in more detail the differences and similarities with the two transformations cited above. Finally, Section 4 presents some conclusions.

\section{Definition of the CGWRPTP}

We first need to introduce some definitions and notations based on previous works, in order to deal with turn penalties:

Let $G=(V, E)$ be a graph similar to the one given in the definition of the CGWRP, with the two costs associated with each edge, one for each direction of traversal. Each pair of edges with a vertex in common, $a=(u, v), b=(v, w) \in E$ has two associated turns at $v$, one based on going from $a$ to $b$ and denoted as $[a b]$, and the other one based on going from $b$ to $a$ and denoted as $[b a]$. Moreover, each edge $e$ incident with $v$ has an associated U-turn at $v$ that will be denoted by [eve]. Each allowed turn in $G$ will have a nonnegative penalty associated with it.

Given $a=(u, v), b=(s, t) \in E$, a $v$-s feasible chain from $a$ to $b$ is an alternating sequence of edges and allowed turns $\left\{a_{1},\left[a_{1} a_{2}\right], a_{2}, \ldots,\left[a_{r-1} a_{r}\right], a_{r},\left[a_{r} b\right]\right\}$, where $a_{1}=a$ in the direction $(u, v)$ and turn $\left[a_{r} b\right]$ belongs to $s$. The cost of a feasible chain is defined as the sum of the costs of the edges it traverses, in their corresponding direction, plus 
the sum of the penalties of the turns it makes. We emphasize the fact that a feasible chain from $a$ to $b$ does not contain edge $b$, and therefore, the cost of this chain does not include the cost of $b$. A $v$-s feasible chain from $a$ to $b$ is closed if $a=b$ and $s \neq v$.

Given $a=(u, v), b=(s, t) \in E$, a shortest (minimum cost) $v$-s feasible chain from $a$ to $b$ will be denoted by s.f.c. $\left(v^{a}, s^{b}\right)$.

Note that in traditional routing problems, the least-cost path for going from vertex $u$ to vertex $v$ and then to vertex $w$, is connecting the shortest path from $u$ to $v$ with the shortest path from $v$ to $w$. But even if these shortest paths take into account turn conditions, the connection of both paths at $v$ can give rise to an unavoidable forbidden turn (U-turn for example). To avoid this last, a feasible chain has been defined such that it begins at a link and ends at a turn. Thus, the connection between two feasible chains at a vertex $v$ is possible only if the first one ends at a turn $[(u, v)(v, w)]$ and the second one begins at the link $(v, w)$, which avoids forbidden turns. Therefore, we cannot use paths between vertices as in standard procedures, which increases the difficulty of modeling the way to satisfy demands at vertices, specially if they contain forbidden turns.

Respect to how to compute shortest feasible chains, the classical method (see e.g. $[7,8])$ is to construct an augmented directed graph where each vertex is duplicated into several vertices such that each allowed turn is replaced by an arc. The drawback of this method is that it is necessary to "expand" all vertices in the graph (required and non-required), and the number of vertices in the augmented graph is $4|E|$. But fortunately, we can use the same idea without physically constructing the augmented graph. If we use suitable vectors, we can use a modification of Dijkstra's algorithm to compute shortest feasible chains directly on the graph, instead of constructing the augmented graph and then computing shortest paths in this graph.

With these concepts we can formally define the Capacitated General Windy Routing Problem with Turn Penalties (CGWRPTP) as follows:

Let $G=(V, E)$ be an undirected graph, $V=\{0,1, \ldots, n\}$ being its set of vertices, where 0 is a depot, and $E$ being the edge set. Each edge $(i, j)$ has two non-negative costs $c_{i j}$ and $c_{j i}$ associated with it, one for each direction of traversal, and each turn 
$[a b]$ has a penalty $p_{[a b]} \geq 0$ associated with it $\left(p_{[a b]}=+\infty\right.$ if turn $[a b]$ is forbidden $)$.

A subset $V_{R}$ of required vertices (including 0 ) and a subset $E_{R}$ of required edges are considered. A positive demand $q_{i}$ is associated with each required vertex $i$ (except for 0) and a positive demand $q_{e}$ is associated with each required edge e. Moreover, a fleet of $k$ vehicles with the same capacity $W$ is available at the depot, where all the turns are allowed with zero penalty.

Find a least-cost set of $k$ closed feasible chains in $G$, one for each vehicle, such that each chain passes through the depot, each demand is served by only one vehicle and the total demand served by each vehicle does not exceed its capacity $W$.

Note that it makes no sense considering turn penalties or forbidden turns at the depot, because in real-world situations, it normally represents a warehouse from which the vehicles begin their journey and to which they return, and they leave from the depot independently of the route they made before. Moreover, these warehouses are usually placed outside the cities with easy access and good road communications.

In the particular case of the CGWRPTP in which $V_{R}=\emptyset$ and the two costs associated with each edge are equal or one of them is infinite, we have the MCARP with turn penalties already studied ([4]). If $k=1$ and the two costs associated with each edge are equal or one of them is infinite, we have the problem studied in [15]. Finally, if all turns are allowed with zero penalty, we have the CGWRP-ed. Then, the problem presented here subsumes all the routing problems with turn penalties previously studied, as well as many of the well-known node and arc routing problems.

\section{Transforming the CGWRPTP into a GVRP}

Let $G$ be a graph where a CGWRPTP is defined, we present in this section a transformation of $G$ into a graph $G^{*}$ where a GVRP is defined. We prove then that the CGWRPTP in $G$ can be transformed in polynomial time into the corresponding GVRP in $G^{*}$, and therefore ([16]), that the CGWRPTP can be transformed into an ACVRP. An example illustrates the complete procedure to make it easier to understand. Finally, we give some comments on the relationship between the transformation presented in 
this paper and other transformations given in previous works.

Let then $G=(V, E)$ be a graph where a CGWRPTP is defined, with required edge set and required vertex set $E_{R}$ and $V_{R}$ respectively. Since in the GVRP the demands are located at the vertices, we will transform graph $G$ into a directed graph $G^{*}=\left(V^{*}, A^{*}\right)$ such that the vertices in $G^{*}$ are related with the required edges and required vertices in $G$. To do this, we partition $V_{R}$ into two subsets, $V_{R_{1}}$ containing the vertices with all allowed zero-penalty turns (including the depot), and $V_{R_{2}}$ containing the vertices with forbidden or positive-penalty turns, in order to construct an intermediate directed graph $G^{\prime}=\left(V^{\prime}, A^{\prime}\right)$ from $G$ as follows:

(1) Initially $G^{\prime}=G$.

(2) Each edge $e \in E$ is replaced in $G^{\prime}$ by two opposite arcs $e_{1}$ and $e_{2}$, each one with the edge cost corresponding to its direction of traversal. Moreover, if $e \in E_{R}$, the two opposite arcs are considered "required", both with demand $q_{e}$. If one of the costs is infinite, the edge is replaced by a single arc $e_{1}$. We have written required between inverted commas because only one of the two generated arcs must be served.

(3) Each $v \in V_{R_{1}}$ gives rise to two vertices $v^{e}$ and $v^{l}$ in $G^{\prime}$, such that $v^{e}$ has only the entering $\operatorname{arcs}$ at $v$ with the same costs and $v^{l}$ has only the leaving arcs from $v$ with the same costs. Add a required $\operatorname{arc} a_{v}=\left(v^{e}, v^{l}\right)$ to $G^{\prime}$ with cost zero and demand $q_{v}$ (demand zero for the arc corresponding to the depot), such that all turns at $v^{e}$ and $v^{l}$ are allowed with zero penalty. It is evident that traversing arc $a_{v}$ in $G^{\prime}$ is equivalent to passing through vertex $v$ in the original graph $G$.

(4) Each $v \in V_{R_{2}}$ is replaced in $G^{\prime}$ by the same number of vertices $v_{i j}$ as that of allowed turns $\left[a_{i} b_{j}\right]$ at $v$, so that each $v_{i j}$ has only one entering arc ( $a_{i}$ with its original cost), one leaving arc $\left(b_{j}\right.$ with its original cost), and its corresponding allowed turn. Then, replace each vertex $v_{i j}$ by two vertices, $v_{i j}^{e}$ and $v_{i j}^{l}$, and add a "required" arc $a_{v_{i j}}=\left(v_{i j}^{e}, v_{i j}^{l}\right)$ between them with cost zero, such that $p_{\left[a_{i} a_{v_{i j}}\right]}=p_{\left[a_{i} b_{j}\right]}$ and $p_{\left[a_{v_{i j}} b_{j}\right]}=0$, i.e. the penalty that was in the turn at $v_{i j}$ is moved to vertex $v_{i j}^{e}$, and all these arcs will have the same demand $q_{v}$.

Note that in (4), if $a_{i}$ is an entering arc at $v, G^{\prime}$ will contain at least as many copies of the entering arc $a_{i}$ as there are allowed turns involving $a_{i}$ at $v$, and the same applies to a leaving $\operatorname{arc} b_{j}$ from $v$. Moreover, as in (2), for each $v \in V_{R_{2}}$, only one of the 
generated arcs $a_{v_{i j}}$ must be served, and traversing only one of these generated arcs $a_{v_{i j}}$ in $G^{\prime}$ is equivalent to passing through vertex $v$ in $G$.

Therefore, we have a directed graph $G^{\prime}=\left(V^{\prime}, A^{\prime}\right)$ such that the subset $A_{R}^{\prime} \subseteq A^{\prime}$ comes from the required vertices and required edges in $G$. From $A_{R}^{\prime}$ we will obtain the partition of the vertex set $V^{*}$ in the graph $G^{*}=\left(V^{*}, A^{*}\right)$ where we will define the GVRP, which we construct as follows:

- For each arc $a_{v} \in A_{R}^{\prime}$ with $v \in V_{R_{1}}$, associate a vertex set $S_{v}=\left\{x_{a_{v}}\right\}$ in $G^{*}$, with demand $q_{v}$.

- For each $v \in V_{R_{2}}$, associate a vertex set $S_{v}$ in $G^{*}$ with demand $q_{v}$ and with as many vertices $x_{a_{v_{i j}}}$ as $\operatorname{arcs} a_{v_{i j}}$ are in $A_{R}^{\prime}$.

- For each pair of opposite required $\operatorname{arcs} e_{1}, e_{2} \in A_{R}^{\prime}$, associate a vertex set $S_{e}$ in $G^{*}$ with demand $q_{e}$ and with as many copies of vertices $x_{e_{1}}$ and $x_{e_{2}}$ as copies of arcs $e_{1}$ and $e_{2}$ respectively appear in $G^{\prime}$. Note that eventually, $S_{e}$ will only contain copies of $e_{1}$ if one traversing cost of $e$ is infinite.

- For each pair of vertices $x_{a}, x_{b} \in V^{*}$ with $x_{a} \in S_{i}, x_{b} \in S_{j}, i \neq j$ being $a=(u, v)$ and $b=(s, t)$, add $\operatorname{arcs}\left(x_{a}, x_{b}\right)$ and $\left(x_{b}, x_{a}\right)$ to $A^{*}$, with the cost of the s.f.c. $\left(v^{a}, s^{b}\right)$ and of the s.f.c. $\left(t^{b}, u^{a}\right)$ respectively in $G^{\prime}$.

- There is no arc between vertices belonging to the same $S_{i}$.

Once we have constructed $G^{*}$, we define a GVRP in this graph with the partition of vertex set $V^{*}$ into the following subsets: $S_{v}$ for all $v \in V_{R_{1}}$ (we will denote the depot subset by $\left.S_{0}=\left\{x_{0}\right\}\right), S_{v}$ for all $v \in V_{R_{2}}$ and $S_{e}$ for all $e \in E_{R}$.

For clarity of the construction of $G^{*}$, we show an example containing the most important steps of this process. Consider the graph given in Figure 2, where vertex 0 represents the depot, there are two required edges $a$ and $b$, with demands 10 and 15 respectively, and a required vertex 1 with demand 15. Moreover, two vehicles are available at the depot, both with capacity 30. Edge costs appear in Figure 2 with their corresponding direction (edges $c, d$ and $f$ are actually arcs), and demands are denoted in italic. All U-turns are considered forbidden except at vertex 0 at which all turns are allowed with penalty zero, and in the rest of the vertices, right turns (according to the representation of the graph) have penalty 1 and left turns have penalty 3 . 


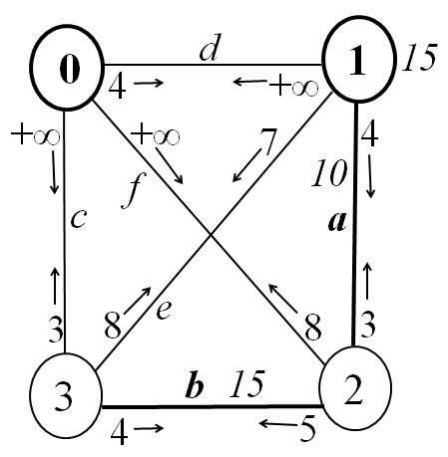

Fig. 2. Graph $G$ with $E_{R}=\{a, b\}$ and $V_{R}=\{0,1\}$.

To construct the intermediate graph $G^{\prime}$ we replace each one of edges $a, b$ and $e$ by two opposite arcs, we replace each one of edges $c, d$ and $f$ by one arc, we replace vertex 0 by the sequence $\left\{0^{e}, a_{0}, 0^{l}\right\}, a_{0}$ with cost zero, and finally, we transform vertex 1, which belongs to $V_{R_{2}}$, into four arcs, as many as allowed turns in it, all of them with cost zero and demand 15. Note that this last step implies that there are copies of arcs $a_{2}, d_{1}$ and $e_{1}$ (see Figure 3 where bold arcs are the "required" ones).

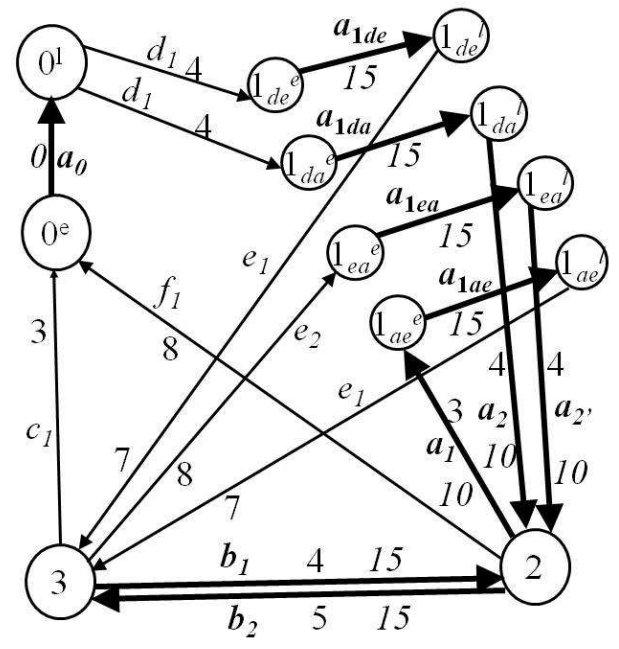

Fig. 3. Intermediate graph $G^{\prime}$.

From $G^{\prime}=\left(V^{\prime}, A^{\prime}\right)$ we construct now the directed graph $G^{*}=\left(V^{*}, A^{*}\right)$ (Figure 4), where $V^{*}$ is given by the following partition:

- A subset $S_{0}$ with a single vertex $x_{0}$ representing the depot, coming from arc $a_{0}$.

- A subset $S_{a}$ with demand 10 and with three vertices $x_{a_{1}}, x_{a_{2}}$ and $x_{a_{2^{\prime}}}$, coming from edge $a=(1,2)$. 
- A subset $S_{b}$ with demand 15 and with two vertices $x_{b_{1}}$ and $x_{b_{2}}$, coming from edge $b=(2,3)$.

- A subset $S_{1}$ with demand 15 and with four vertices $x_{a_{1 d e}}, x_{a_{1 d a}}, x_{a_{1 e a}}$ and $x_{a_{1 a e}}$, coming from vertex 1 in $V_{R_{2}}$.

For simplicity, in Figure 4 each pair of $\operatorname{arcs}\left(x_{r}, x_{t}\right)$ and $\left(x_{t}, x_{r}\right)$ with $x_{r} \in S_{i}, x_{t} \in S_{j}$ and $i \neq j$, has been drawn as a line with two arrow heads, one at each end, and the arc costs (normally different for each direction) have been omitted.

From now on, if $R$ represents a route or a set of routes in a defined graph $G\left(G^{\prime}\right)$ $\left(G^{*}\right)(\hat{G})$, we will denote by $c(R)\left(c^{\prime}(R)\right)\left(c^{*}(R)\right)(\hat{c}(R))$ the cost of $R$ in $G\left(G^{\prime}\right)\left(G^{*}\right)$ $(\hat{G})$ according to the corresponding cost definition.

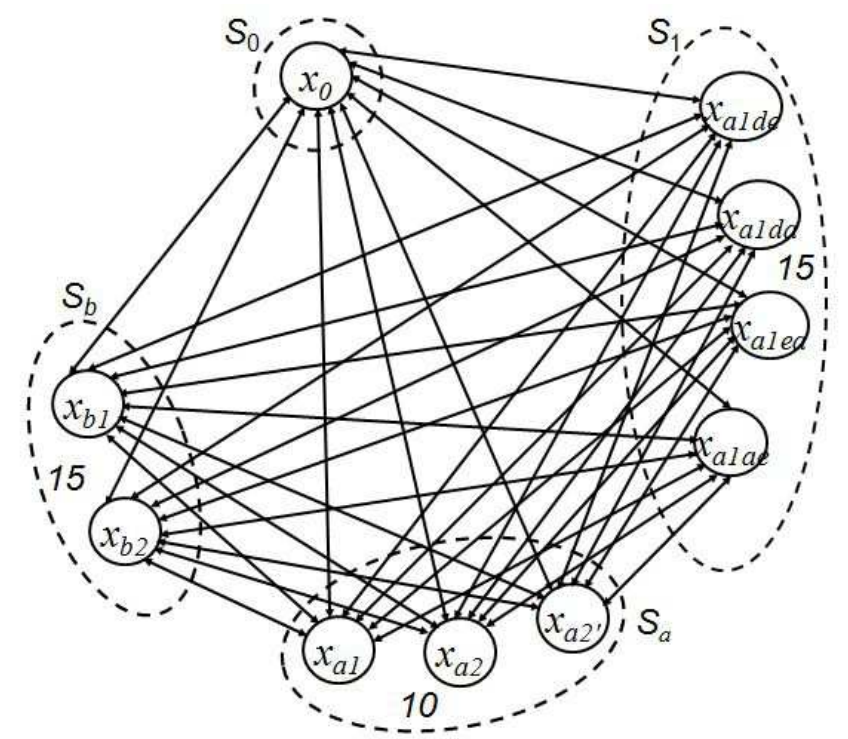

Fig. 4. Directed graph $G^{*}$.

Theorem 1 A CGWRPTP defined in $G$ can be transformed in polynomial time into the corresponding GVRP defined in $G^{*}$.

Proof. Let $B=\left\{T_{i}\right\}_{i=1}^{k}$ be a set of $k$ feasible closed chains in $G$ corresponding to a solution of the CGWRPTP. By the construction of $G^{\prime}$ we can associate with $B$ a set $B^{\prime}=\left\{T_{i}^{\prime}\right\}_{i=1}^{k}$ of $k$ feasible closed chains in $G^{\prime}$ such that for all $i \in\{1, \ldots, k\}$ we have: $T_{i}^{\prime}$ traverses arc $a_{0}$ (corresponding to the depot in $G$ ); $T_{i}$ passes through a vertex $v \in V_{R_{1}}$ iff $T_{i}^{\prime}$ traverses arc $a_{v} \in A_{R}^{\prime} ; T_{i}$ passes through a vertex $v \in V_{R_{2}}$ iff $T_{i}^{\prime}$ traverses an $\operatorname{arc} a_{v_{i j}} ; T_{i}$ traverses edge $e \in E$ iff $T_{i}^{\prime}$ traverses a copy of arc $e_{1}$ or a copy of arc $e_{2}$ 
in $A_{R}^{\prime}$; and $T_{i}^{\prime}$ has the same cost as $T_{i}$. Moreover, we will suppose that if $T_{i}$ satisfies demand at $v \in V_{R_{1}}\left(v \in V_{R_{2}}\right)\left(e \in E_{R}\right)$, then $T_{i}^{\prime}$ satisfies the demand located at $a_{v}$ (one and only one arc $a_{v_{i j}}$ ) (one and only one copy of $e_{1}$ or $e_{2}$ in $G^{\prime}$ ).

Then we have that $\forall i \in\{1, \ldots, k\}, T_{i}^{\prime}$ is a feasible closed chain in $G^{\prime}$ that traverses $a_{0}$, satisfies the same demands as $T_{i}$ and has the same cost as $T_{i}$.

For each $i \in\{1, \ldots, k\}$, from $T_{i}^{\prime}$ we construct now a route $C_{i}^{B}$ in $G^{*}$ as follows: if $T_{i}^{\prime}$ satisfies, in this order, the demands $q_{j_{1}}, q_{j_{2}}, \ldots, q_{j_{m_{i}}}, C_{i}^{B}$ is a route in $G^{*}$ that visits, in this order, the vertex sets $S_{0}, S_{j_{1}}, S_{j_{2}}, \ldots, S_{j_{m_{i}}}, S_{0}$, and $\forall t \in\left\{1, \ldots, m_{i}\right\}, C_{i}^{B}$ visits only the vertex of $S_{t}$ coming from the arc in $G^{\prime}$ served by $T_{i}^{\prime}$.

The set $L^{B}=\left\{C_{i}^{B}\right\}_{i=1}^{k}$ is then a solution of the GVRP in $G^{*}$, and it is easy to see that the cost $c^{*}\left(L^{B}\right)$ is less than or equal to $c(B)$.

On the other hand, let $L=\left\{C_{i}\right\}_{i=1}^{k}$ be a set of $k$ routes corresponding to a solution of the GVRP in $G^{*}$. For each $i \in\{1, \ldots, k\}$, from $C_{i}$ we construct a feasible closed chain $T_{i}^{\prime L}$ in $G^{\prime}$ as follows:

Let $\left(x_{a}, x_{b}\right)$ be a generic arc of $C_{i}, a=(u, v)$ and $b=(w, r)$ being the arcs in $A_{R}^{\prime}$ from which vertices $x_{a}$ and $x_{b}$ come, respectively. Arc $\left(x_{a}, x_{b}\right)$ will give rise in $T_{i}^{L}$ to the s.f.c. $\left(v^{a}, w^{b}\right)$, such that $T_{i}^{L}$ satisfies the demand at $a$ (the same as the one in $x_{a}$ ) and $b$ (the same as the one in $x_{b}$ ). Note that in this way, $T_{i}^{\prime L}$ has the same cost as $C_{i}$ and satisfies the same demands as $C_{i}$.

From the set $B^{\prime L}=\left\{T_{i}^{L}\right\}_{i=1}^{k}$ of $k$ feasible closed chains in $G^{\prime}$, we construct now a set $B^{L}=\left\{T_{i}^{L}\right\}_{i=1}^{k}$ of $k$ feasible closed chains in $G$ as follows:

- "Contract" each sequence in $T_{i}^{L}$ of the form $\left(u, v^{e}\right)\left(v^{e}, v^{l}\right)\left(v^{l}, w\right)$ with $a_{v}=\left(v^{e}, v^{l}\right)$ if $v \in V_{R_{1}}$ by $(u, v)(v, w)$ in $T_{i}^{L}$.

- "Contract" each sequence in $T_{i}^{L L}$ of the form $\left(u, v_{i j}^{e}\right)\left(v_{i j}^{e}, v_{i j}^{l}\right)\left(v_{i j}^{l}, w\right)$ with $a_{v_{i j}}=$ $\left(v_{i j}^{e}, v_{i j}^{l}\right)$ if $v \in V_{R_{2}}$ by $(u, v)(v, w)$ in $T_{i}^{L}$.

- If $T_{i}^{L}$ traverses a copy of $\operatorname{arc} e_{1}$ or a copy of $\operatorname{arc} e_{2}$ in $G^{\prime}$, with $e \in E$, replace this copy in $T_{i}^{L}$ by edge $e$.

- Any other link or turn in $T_{i}^{L}$ is replaced by itself in $T_{i}^{L}$.

- Demand at $v \in V_{R_{1}}\left(v \in V_{R_{2}}\right)\left(e \in E_{R}\right)$ is assigned to $T_{i}^{L}$ iff $T_{i}^{L}$ satisfies demand located at $a_{v}$ (one and only one arc $a_{v_{i j}}$ ) (one and only one copy of $e_{1}$ or $e_{2}$ in $G^{\prime}$ ). 
It is evident that $B^{L}=\left\{T_{i}^{L}\right\}_{i=1}^{k}$ is a solution of the CGWRPTP in $G$ with $c\left(B^{L}\right)=$ $c^{*}(L)$, this last due to the fact that for all $i, c\left(T_{i}^{L}\right)=c^{\prime}\left(T_{i}^{\prime L}\right)$.

Let then $L^{o p t}$ be an optimal GVRP solution in $G^{*}$ and let $B^{L^{o p t}}$ be the CGWRPTP solution in $G$ obtained from $L^{\text {opt }}$ as described above, for each CGWRPTP solution $B$ in $G$ we have that $c(B) \geq c^{*}\left(L^{B}\right) \geq c^{*}\left(L^{o p t}\right)=c\left(B^{L^{o p t}}\right)$, and therefore, $B^{L^{o p t}}$ is an optimal CGWRPTP solution in $G$. I

Once we have transformed the CGWRPTP into a GVRP defined in $G^{*}$, to solve the GVRP in $G^{*}$, from this graph we can construct a digraph $\hat{G}$ where an ACVRP is defined. We omit the construction of $\hat{G}$ and the transformation of an ACVRP solution in $\hat{G}$ into a GVRP solution in $G^{*}$ because they can be obtained from [16]. Note only that an optimal ACVRP solution $H_{\text {opt }}$ in $\hat{G}$, gives rise to an optimal GVRP solution $L_{\text {opt }}$ in $G^{*}$ with cost $c^{*}\left(L_{\text {opt }}\right)=\hat{c}\left(H_{\text {opt }}\right)-M(m+k), M$ being a large positive number and $m$ being the number of vertex subsets in $G^{*}$, the depot not included.

In our example, from graph $G^{*}$ (Figure 4) we define the ACVRP in the digraph $\hat{G}$ (Figure 5) where, for simplicity again, the pairs of $\operatorname{arcs}\left(x_{r}, x_{t}\right)$ and $\left(x_{t}, x_{r}\right)$ with $x_{r} \in \hat{S}_{i}, x_{t} \in \hat{S}_{j}$ and $i \neq j$ have been drawn as lines with two arrow heads, one at each end, and the arc costs have been omitted. Figure 5 shows the cost zero "intraset" arcs and the demand assigned to each vertex. For example, edge $a$ with demand 10 in $G$, has associated the set $S_{a}$ in $G^{*}$ with three vertices that in $\hat{G}$ have demands 4,3 and 3 respectively.

Figure 6 shows the optimal solution $H$ to the ACVRP in $\hat{G}$; it consists of two routes: $H_{1}=\left(x_{0}, x_{b_{2}}, x_{b_{1}}, x_{0}\right)$ with cost $19+2 M$ and servicing the demand $q_{b}=15$, and $H_{2}=\left(x_{0}, x_{a 1_{d a}}, x_{a 1_{e a}}, x_{a 1_{a e}}, x_{a 1_{d e}}, x_{a_{2}}, x_{a_{1}}, x_{a_{2^{\prime}}}, x_{0}\right)$ with cost $18+3 M$ and servicing the sum of demands $q_{1}+q_{a}=25$.

This optimal solution $H$ in $\hat{G}$ gives rise to the optimal solution $L$ in $G^{*}$ shown in Figure 7, with total cost $c^{*}(L)=\hat{c}(H)-5 M=37$, and that consists of routes $C_{1}=\left(x_{0}, x_{b_{2}}, x_{0}\right)$ with cost 19 and $C_{2}=\left(x_{0}, x_{a 1_{d a}}, x_{a_{2}}, x_{0}\right)$ with cost 18.

Finally, following the proof of Theorem 1 and with the aid of Figures 2 and 3, from these two routes in $G^{*}$ we can obtain the optimal solution to the CGWRPTP in $G$ (Figure 8). For simplicity we will not write the intermediate turns of a feasible chain. For example, the feasible chain $\{a,[a b], b,[b, c]\}$ will be written as $\{a, b,[b, c]\}$. 
From $C_{1}=\left(x_{0}, x_{b_{2}}, x_{0}\right)$ in $G^{*}$ we obtain the feasible closed chain

$T_{1}=\{(0,1),(1,2),(2,3),(3,0)[(3,0),(0,1)]\}$ in $G$, containing the depot node and satisfying the demand of edge $b(15)$, and from $C_{2}=\left(x_{0}, x_{a 1_{d a}}, x_{a_{2}}, x_{0}\right)$ in $G^{*}$ we obtain the feasible closed chain $T_{2}=\{(0,1),(1,2),(2,0)[(2,0),(0,1)]\}$ in $G$, containing the depot node and satisfying the demands of vertex 1 (15) and edge $a$ (10).

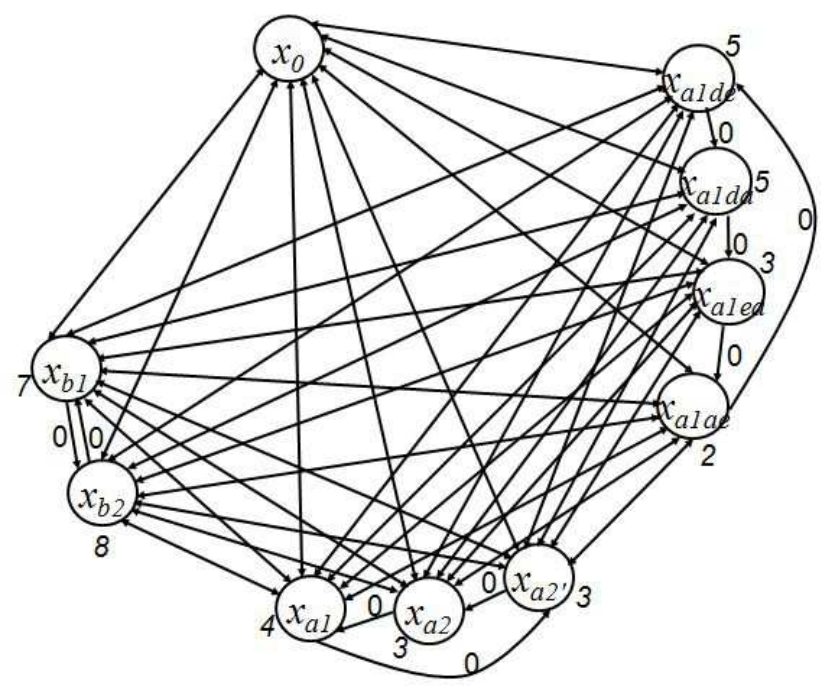

Fig. 5. Directed graph $\hat{G}$ associated with $G^{*}$.

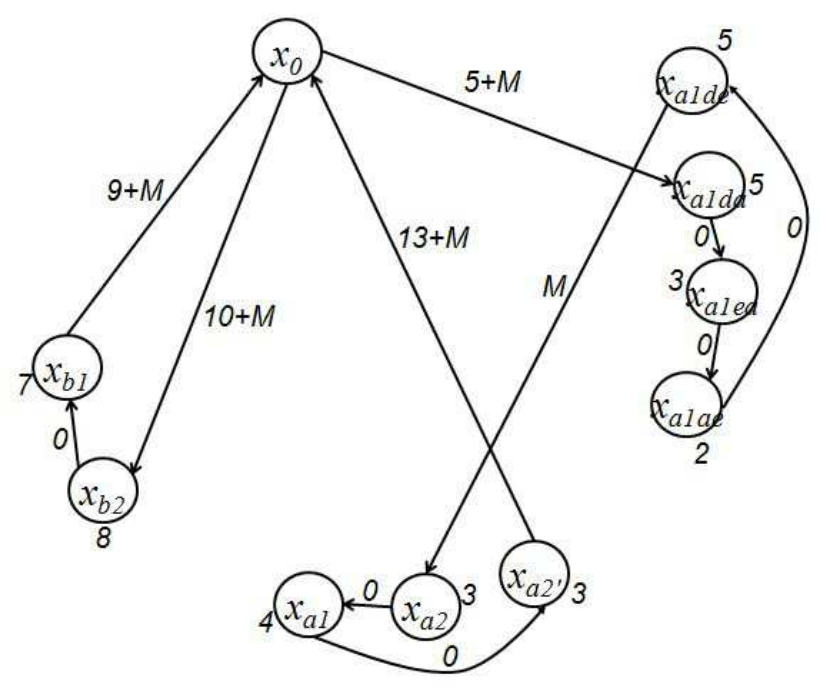

Fig. 6. Optimal solution to the ACVRP in $\hat{G}$.

Next we make some comments on the relationship between the transformation presented in this paper and other transformations given in previous works.

Our transformation can be considered an extension to the windy and multivehicle case of the transformation into an Asymmetric TSP of the single vehicle routing prob- 
lem studied in [15]. By comparing the whole process of both transformations, it is clear that the existence of more than one vehicle, depot, demands and capacities, requires a more elaborated and complex proof that the transformation is useful, which is based on results about two multivehicle routing problems, the ACVRP and the GVRP.

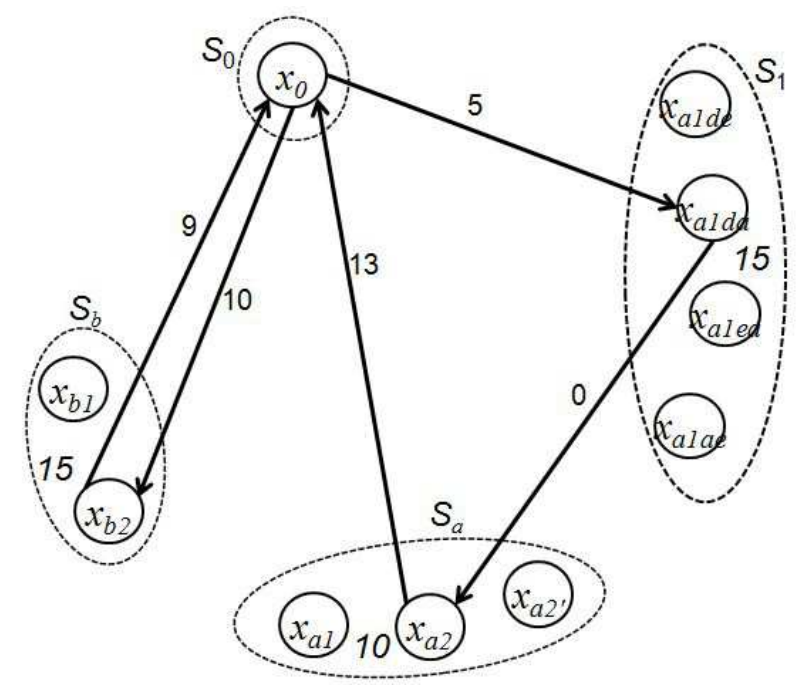

Fig. 7. Optimal solution to the GVRP in $G^{*}$.

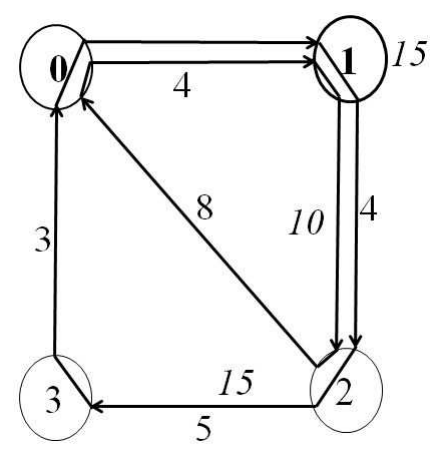

Fig. 8. Optimal solution to the CGWRPTP in $G$.

However, if we put our attention on the structure of the transformed graph, except for the existence of demands and a depot node, the structure of the graphs are very similar in both procedures. The main two differences, which are interrelated, are:

1) In [15], due to the fact that only one vehicle is used, it was assumed that a required vertex is not incident with a required link; otherwise, traversing the required link involves crossing the required vertex, and then the restriction "required" is redundant for that vertex. This assumption implies that copies of required arcs in $G$ will never appear in $G^{\prime}$. But when $k>1$, if a required vertex is incident with a required link, 
in order to meet the capacity constraints or to minimize the total cost, the demand of the required link and the demand of the required vertex can be served by different vehicles. Therefore, in the multivehicle case we can not assume that a required vertex is not incident with a required link. This implies that in $G^{\prime}$ copies of a required link may appear, depending on the number of allowed turns involving this link at the required vertex, which affects to the size of $G^{*}$ and to the complexity of the transformation. This happens in our example (see Figure 2), where required edge $a$ is incident with required vertex 1 . In this case $G^{\prime}$ contains two copies of arc $a_{2}$ (see Figure 3 ) and then, $S_{a}$ contains three vertices in $G^{*}$ (see Figure 4).

2) In [15] each edge $e=(u, v)$ always gives rise to two clusters $\left\{u_{e}\right\}$ and $\left\{v_{e}\right\}$ (both with a single vertex), and two $\operatorname{arcs}\left(u_{e}, v_{e}\right)$ and $\left(v_{e}, u_{e}\right)$, both with cost $-M, M$ being a very large positive number (see Figure 8 in [15]). Each direction of $e$ is associated with one of these arcs, and due to their costs, we have the guarantee that one and only one of these arcs will be in the optimal solution. In our transformation we do not use arcs with cost $-M$ in $G^{*}$, which is better for computational reasons. In the single vehicle particular case, for each required edge $e=(u, v)$, our transformation gives rise to a cluster with two vertices $\left\{u_{e}, v_{e}\right\}$, such that each direction of $e$ is associated with one of the vertices in the cluster, not with an arc. Therefore, if we apply our procedure to a single vehicle case, the transformed graph have the same number of vertices than the transformed graph given in [15], but all arcs associated with required edges have different costs in both transformed graphs.

On the other hand, if we apply the transformation presented here to the CGWRPed (the particular case of the CGWRPTP in which all turns are allowed with zero penalty), the graph $G^{*}$ where the GVRP is defined is the same as that obtained in [1], except for the arcs costs. This is due to the fact that we compute shortest feasible chains between arcs, whose costs, by definition ([8]), contain the cost of the initial arc but not the cost of the final arc, whereas in the procedure shown in [1], the arc costs defined for the GVRP contain the cost of the final arc but not the cost of the initial arc. Note that despite the difference in the arc costs, the GVRP solution is the same in both procedures because each cycle has the same cost in both graphs where the GVRP is defined. Therefore, somehow, our transformation can also be considered as 
an extension to turn penalties and forbidden turns of the transformation given in [1].

Finally, note that one can think of an alternative to the procedure described here to transform the CGWRPTP into a GVRP. This alternative may consist of constructing the augmented graph mentioned in Section 2 (to avoid turn penalties) and then transforming this augmented graph into another one where the GVRP can be applied. However, none of the previous papers solving routing problems with turn penalties through a transformation obtains the transformed graph from the augmented graph. It seems more efficient to compute shortest feasible chains directly on the graph and construct the transformed graph from the original one, instead of constructing the augmented graph and then to compute shortest paths in this graph and obtain the transformed graph from it. Moreover, if the number of required elements is small compared to the total number of elements, as it is normal in problems inside big cities, the option of the augmented graph seems much less attractive, because it needs to expand all vertices in the graph (required and non-required), as we commented in Section 2.

\section{Conclusion}

This paper deals with a problem that generalizes most of the existing node and arc routing problems and all their studied extensions that take into account turn penalties and forbidden turns. It provides a way to solve this general problem by transforming it into an ACVRP. We are convinced that research on vehicle routing problems will take into account more and more the existence of turn penalties to tackle real-life routing problems inside big cities. In this way, the aim of this paper is to provide a tool to help future researchers to study the efficiency of specific heuristics to solve vehicle routing problems with turn penalties that could be modeled as particular cases of the problem presented here. For example, they could compare the results obtained with their specific heuristics with those obtained (through our transformation) with existing or future competitive ACVRP heuristic algorithms. 


\section{Acknowledgements}

This work has been partially supported by the Ministerio de Educación y Ciencia of Spain (project TIN2008-06441-C02-01). We are also indebted to the two anonymous referees for their valuable comments.

\section{References}

[1] R. Baldacci, E. Bartolini, G. Laporte. Some applications of the generalized vehicle routing problem, Journal of the Operational Research Society 61 (2010) 1072-1077.

[2] J. Bautista, J. Pereira. Ant algorithms for urban waste collection routing, Lecture Notes in Computer Science 3172 (2004), 3020-3033.

[3] J. Bautista, E. Fernández, J. Pereira. Solving an urban waste collection problem using ants heuristics, Computers \& Operations Research 35 (2008) 302-309.

[4] J.M. Belenguer, E. Benavent, P. Lacomme, C. Prins. Lower and upper bounds for the mixed capacitated arc routing problem, Computers \& Operations Research 33 (2006) 3363-3383.

[5] E. Benavent, D. Soler. The directed rural postman problem with turn penalties, Transportation Science 33 (1999) 408-418.

[6] L. Bodin, G. Fagin, R. Welebny, J. Greenberg. The design of a computerized sanitation vehicle routing and scheduling for the town of Oyster Bay, New York, Computers \& Operations Research 16 (1989) 45-54.

[7] J. Clossey, G. Laporte, P. Soriano. Solving arc routing problems with turn penalties, Journal of the Operational Research Society 52 (2001) 433-439.

[8] A. Corberán, R. Martí, E. Martínez, D. Soler. The rural postman problem on mixed graphs with turn penalties, Computers \& Operations Research 29 (2002) 887-903.

[9] R. De Franceschi, M. Fischetti, P. Toth. A new ILP-based refinement heuristic for vehicle routing problems, Math Programming Ser B 105 (2006) 471-499.

[10] M. Fischetti, P. Toth, D. Vigo. A branch-and bound algorithm for the capacitated vehicle routing problem on directed graphs, Operations Research 42 (1994) 846-859. 
[11] G. Ghiani, G. Improta. An efficient transformation of the generalized vehicle routing problem, European Journal of Operational Research 122 (2000) 11-17.

[12] N. Perrier, A. Langevin, C.V. Amaya. Vehicle routing for urban snow plowing operations, Transportation Science 42 (2008) 44-56.

[13] A. Pessoa, M. Poggi, E. Uchoa. Robust branch-cut-and-price algorithms for vehicle routing problems, Route 2007 (International Workshop on Vehicle Routing and Transportation) Jekyll Island, Georgia (2007).

[14] S. Roy, J.M. Rousseau. The capacitated canadian postman problem, INFOR 27 (1989) 58-73.

[15] D. Soler, E. Martínez, J.C. Micó. A transformation for the mixed general routing problem with turn penalties, Journal of the Operational Research Society 59 (2008) 540-547.

[16] D. Soler, J. Albiach, E. Martínez. A way to optimally solve a time-dependent vehicle routing problem with time windows, Operations Research Letters 37 (2009) 37-42.

[17] D. Vigo. A heuristic algorithm for the asymmetric capacitated vehicle routing problem. European Journal of Operational Research 89 (1996) 108-126. 